Archive for

Organic Chemistry

Arkivoc 2020, part vii, $180-191$

\title{
The reactivity of Oxone towards 4,6-di(cycloamino)-1,3-phenylenediamines: synthesis of spirocyclic oxetane ring-fused imidazobenzimidazoles
}

\author{
Darren Conboy and Fawaz Aldabbagh
}

Department of Pharmacy, School of Life Sciences, Pharmacy and Chemistry, Kingston University, Penrhyn Road, Kingston upon Thames, KT1 2EE, U.K.

Email: f.aldabbagh@kingston.ac.uk

Dedicated to Professor Jan Bergman on the occasion of his $80^{\text {th }}$ birthday

Received 04-25-2020

Accepted 06-29-2020

Published on line $07-31-2020$

\section{Abstract}

Spirocyclic oxetane ring-fused imidazo[4,5-f] benzimidazole and imidazo[5,4-f]benzimidazole are reported. Oxone-mediated ring-closures to give imidazobenzimidazoles require acid and the functionalization of the 4,6di(cycloamino)-1,3-phenylenediamine to the anilides. This is in contrast to benzimidazole forming oxidative cyclizations, which use 2-(cycloamino)anilines and require no acid. New evidence for $N$-oxide and nitrosointermediates in respective imidazobenzimidazole and benzimidazole forming reactions is provided.

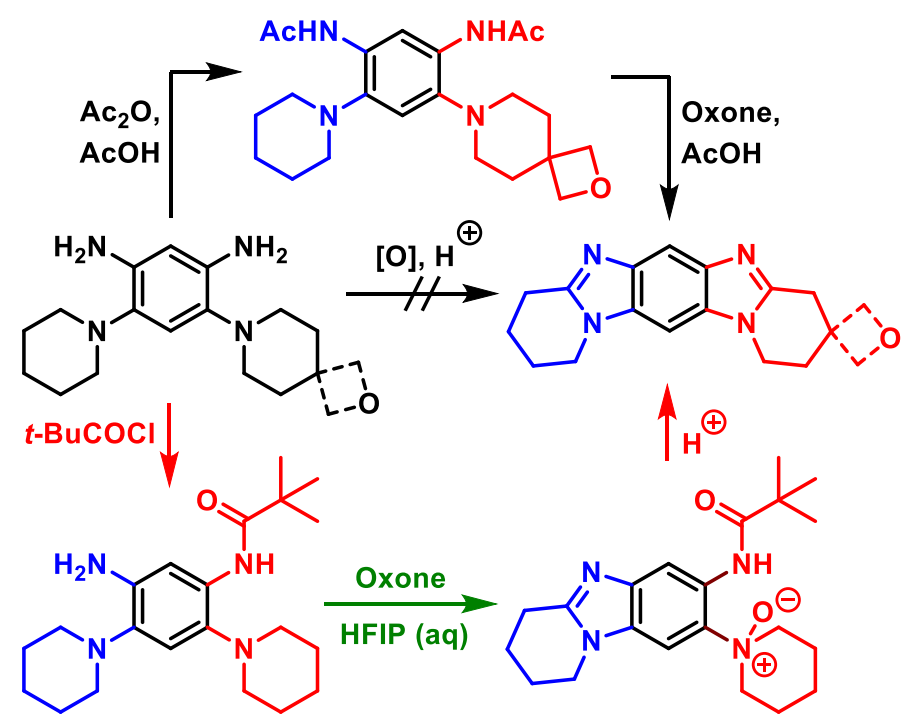

Keywords: Anilide, oxidative cyclization, benzimidazole, heterocycle, HFIP 


\section{Introduction}

Imidazobenzimidazoles exist in a $[4,5-f]$ and $[5,4-f]$ arrangement and are scaffolds at the core of antitumor agents. ${ }^{1-5} \mathrm{~A}$ valuable strategy for the discovery of chemotherapeutics is the targeting of proteins overexpressed in solid tumors, such as $\mathrm{NAD}(\mathrm{P}) \mathrm{H}$ :quinone oxidoreductase 1 (NQO1, also known as DT-diaphorase). ${ }^{6}$ For iminoquinone derivatives of imidazo[5,4-f]benzimidazoles, a hydrogen bonding acceptor improves binding at the NQO1 active site. ${ }^{5}$ Oxetane is a robust hydrogen bonding alternative to carbonyl functionalities and a polar analogue of the gem-dimethyl group. ${ }^{7,8}$ Spirocyclic oxetane $\mathbf{1}$ was fused onto benzimidazole via oxidative cyclizations of 2-(2-oxa-7-azaspiro[3.5]nonan-7-yl)aniline $\mathbf{2 a}$ and acetamide analogue $\mathbf{2 b}$ using $\mathrm{H}_{2} \mathrm{O}_{2}$ with $\mathrm{HI}$ and Oxone (potassium peroxymonosulfate) in formic acid to give $\mathbf{3 a}$ and $\mathbf{3} \mathbf{b}$ respectively (Scheme 1 ) ${ }^{9,10}$ In this article, our synthetic targets are spirocyclic oxetane ring-fused isomers, imidazo[4,5-f]benzimidazole 4 and imidazo[5,4-f]benzimidazole 5 (Figure 1).

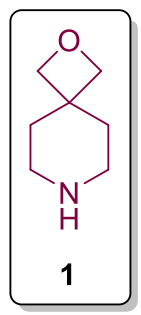

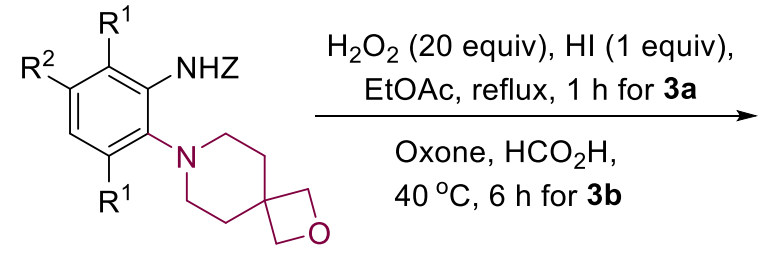

2a: $Z=H, R^{1}=O M e, R^{2}=H$

2b: $Z=A c, R^{1}=H, R^{2}=B r$

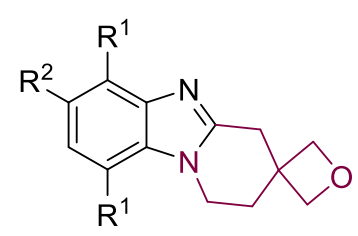

$3 a, 74 \%$

3b, $74 \%$

Scheme 1. Reported preparations of benzimidazoles with ring-fused spirocyclic oxetane. ${ }^{9,10}$

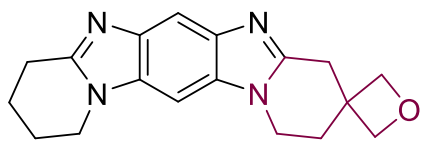

imidazo[4,5- $f$ benzimidazole 4

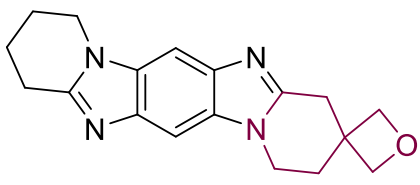

imidazo[5,4-f]benzimidazole 5

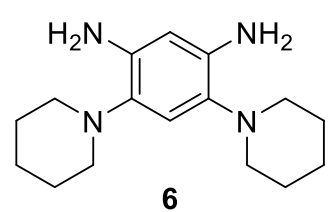

6

Figure 1. Target imidazobenzimidazoles and the investigated diamine.

The fundamental advantage of using the Oxone protocol over earlier reported radical cyclization methods, ${ }^{3}$ is the ability to synthesize imidazobenzimidazoles (e.g. 7) containing two different fused rings (Scheme 2). ${ }^{4}$ For cyclization, acid has to be present, and $m$-CPBA in acetone gave the diamine $N$-oxide 8 , as the sole product. Treatment of diamine- $N$-oxide $\mathbf{8}$ with formic acid in the absence of an external oxidant gave ringfused benzimidazole $\mathbf{9}$, and a mechanism for oxidative cyclization through the internal conjugated system of $\mathbf{8}$ was provided. ${ }^{4}$ The use of Oxone for the preparation of these fanciful imidazobenzimidazole scaffolds is reminiscent of early work by Spiegel and Kaufmann, who utilized Caro's acid (peroxysulfuric acid) to cyclize 5nitro-2-(piperidin-1-yl)aniline to give benzimidazole. ${ }^{11}$ Our group has also shown that $\mathrm{H}_{2} \mathrm{O}_{2}$ can be utilized in the absence of acid for some cyclizations of 2 -(cycloamino)anilines, ${ }^{12}$ and with $\mathrm{HX}(\mathrm{X}=\mathrm{Cl}$ and $\mathrm{Br})$ to give selectively halogenated ring-fused benzimidazoles, ${ }^{13}$ and benzimidazolequinones. ${ }^{14}$ In this article, we examine the reactivity of Oxone towards anilide derivatives of 4,6-di(piperidin-1-yl)-1,3-phenylenediamine (6) (Figure 1), as part of studies towards the preparation of spirocyclic oxetane ring-fused imidazobenzimidazoles $\mathbf{4}$ and $\mathbf{5}$. 

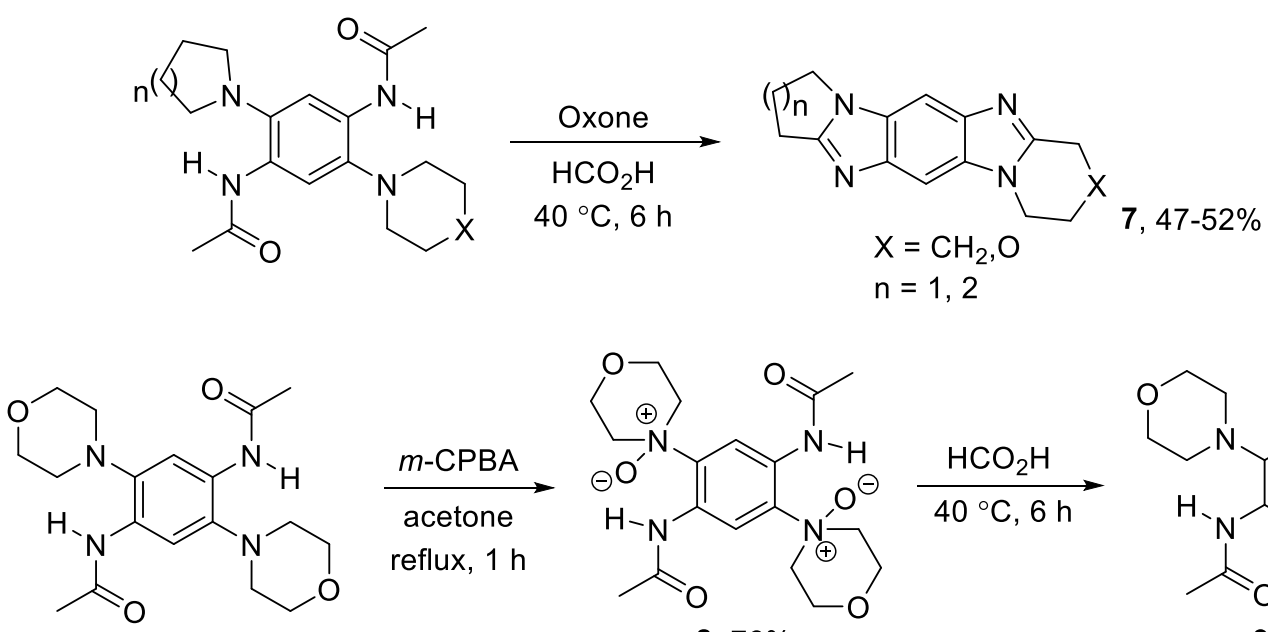

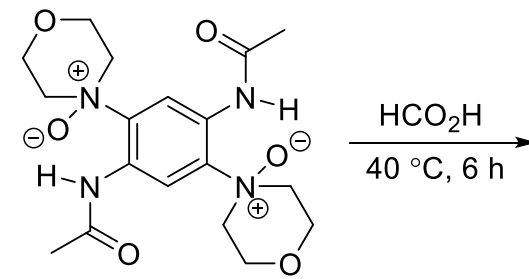

8, $76 \%$

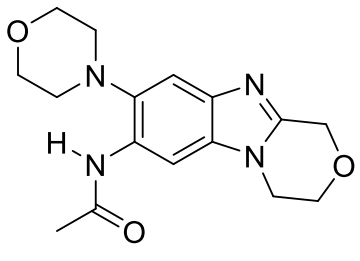

9, $80 \%$

Scheme 2. Reported oxidative cyclizations and evidence of diamine $N$-oxide intermediate. ${ }^{4}$

\section{Results and Discussion}

Treating diamine 6 with Oxone in acid did not give imidazo[4,5-f]benzimidazole, but led to intractable mixtures. This is in line with our findings in Scheme $2,{ }^{4}$ where decreasing the electron-density by acetylating at the primary amines of 6 was necessary. This led us to investigate the effect of Oxone on the mono-anilide derivative of $\mathbf{6}$. Slow addition of sterically hindered pivaloyl chloride was necessary in order to minimize reaction at both amines of 6 , which gave the desired $N$-[5-amino-2,4-di(piperidin-1-yl)phenyl]-2,2dimethylpropanamide (10) and $N, N^{\prime}$-[4,6-di(piperidin-1-yl)-1,3-phenylene]bis(2,2-dimethylpropanamide) (11) in 61 and $14 \%$ yield respectively (Scheme 3 ).

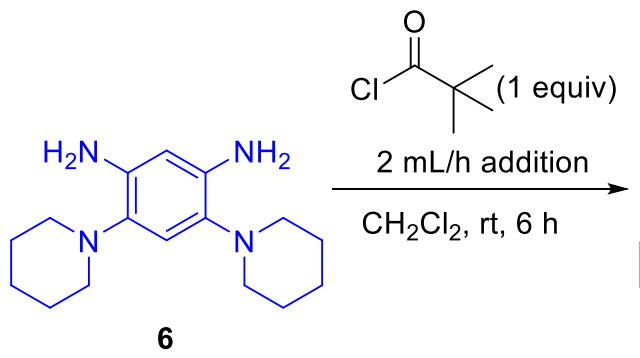

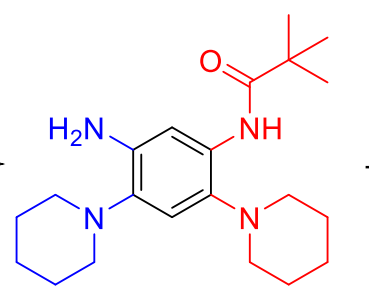

$10,61 \%$

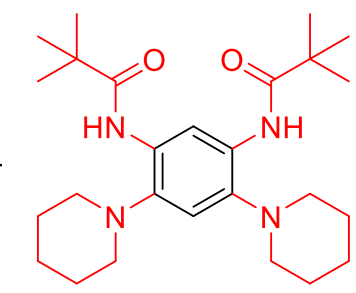

$11,14 \%$

Scheme 3. Functionalizing 4,6-di(piperidin-1-yl)-1,3-phenylenediamine (6) with pivaloyl chloride.

Reaction of amine-anilide $\mathbf{1 0}$ with Oxone in a $10 \%$ aqueous solution of hexafluoroisopropanol (HFIP) gave the intriguing adduct 12 in 58\% yield, resulting from cyclization and $N$-oxide formation at the amine and anilide parts respectively (Scheme 4). Mono-cyclized adduct 13 was also formed in $29 \%$ yield due to insufficient oxidation to 12 . The amide singlet is shifted to $14 \mathrm{ppm}$ in the ${ }^{1} \mathrm{H}-\mathrm{NMR}$ spectrum of 12 , consistent with strong hydrogen bonding to the $N$-oxide (amide- $\mathrm{NH}$ at $\approx 9.3 \mathrm{ppm}$ in compounds 10,11 and 13 ), a trait observed for related $\mathrm{N}$-oxides, including diacetamide $8 .{ }^{4}$ The use of HFIP as reaction solvent enabled the solvation of hydrogen-bonding acceptors, including 10 and/or intermediates. ${ }^{15}$ Treating 12 with 
methanesulfonic acid (MsOH) gave the imidazo[4,5-f] benzimidazole 14 in 42\%, ${ }^{3,16}$ and benzimidazole-anilide 13 in 44\% yield, in which presumably the $N$-oxide part of 12 allowed intermolecular oxidative aromatization. ${ }^{4}$

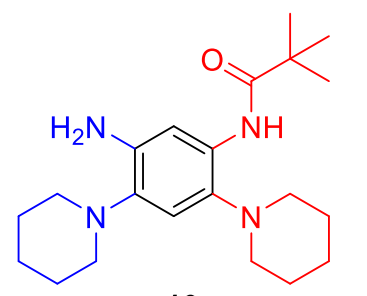

10

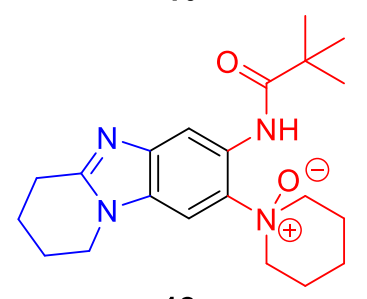

12

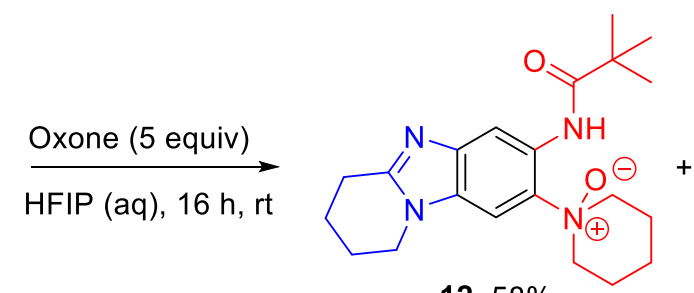

$12,58 \%$

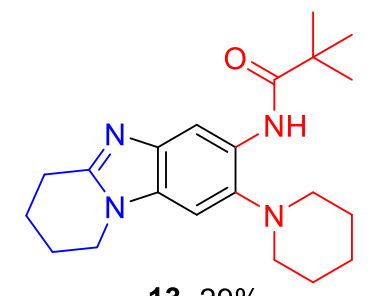

$13,29 \%$

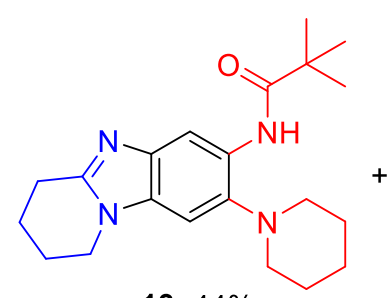

$13,44 \%$

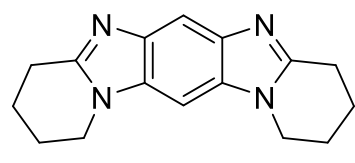

$14,42 \%$

Scheme 4. Reactions of anilide derivatives of 6.

The formation of $\mathbf{1 2}$ highlights the difference in mechanism for oxidative cyclization of 2(cycloamino)anilines and 2-(cycloamino)anilides (Scheme 4). Literature advocates a nitroso intermediate for the formation of benzimidazoles from 2-(cycloamino)anilines, ${ }^{9,17-19}$ although the o-nitroso-tert-aniline has never been isolated. Most recently, we observed the 4-(2-nitrosophenyl)morpholine intermediate by GC-MS from the oxidative cyclization to the ring-fused benzimidazole, which under certain conditions underwent displacement of oxazine to give 1,4,6,9-tetramethoxyphenazine. ${ }^{9}$ Evidence for the o-nitroso-tert-aniline intermediate $\mathbf{1 6}$ is now provided from the reaction of 2-(morpholin-4-yl)aniline (15) with Oxone in a $\mathrm{CH}_{2} \mathrm{Cl}_{2}: \mathrm{H}_{2} \mathrm{O}$ mixture (Scheme 5). Quenching this reaction at short times of $30 \mathrm{~s}$ and 2 min gave mostly 4-(2nitrophenyl)morpholine (18), which indicates formation of intermediate 16 and advantageous air-oxidation. After 18 and $40 \mathrm{~h}$, it is apparent from the ${ }^{1} \mathrm{H}$ NMR spectra that the morpholine signals at 3.06 and $3.85 \mathrm{ppm}$ for 4-(2-nitrophenyl)morpholine (18) have been replaced by those for benzimidazole 17 at 4.20 and 5.05 ppm.

The above mechanistic work clarified the requirement for diacetamides $\mathbf{2 1}$ and $\mathbf{2 4}$ for the respective formation of imidazobenzimidazoles $\mathbf{4}$ and $\mathbf{5}$, negating direct oxidative cyclizations from phenylenediamines 20 and $\mathbf{2 3}$ (Scheme 6). Moreover treatment of diamines $\mathbf{2 0}$ and $\mathbf{2 3}$ with Oxone in HFIP under the conditions of Scheme 4 gave an intractable mixture. Syntheses began by nucleophilic aromatic substitution $\left(S_{N} A r\right)$ onto 1,5difluoro-2,4-dinitrobenzene and 1,4-difluoro-2,5-dinitrobenzene with bis(2-oxa-7-azaspiro[3.5]nonan-7-ium) ethanedioate (oxalate salt of 1 ), followed by $S_{N} A r$ with the stronger nucleophile, piperidine to give the respective unsymmetrically substituted dinitrobenzenes 19 and 22 in 85 and 79\% yield. Hydrogenation to the phenylenediamines $\mathbf{2 0}$ and $\mathbf{2 3}$ occurred in 91 and $88 \%$ yield, and reaction with acetic anhydride gave the cyclization precursors 21 and 24 in 84 and 86\% yield respectively. Oxidative cyclizations of 21 and 24 with Oxone ( 6 equiv) in acetic acid gave imidazo[4,5-f]benzimidazole 4 and imidazo[5,4- $f$ benzimidazole 5 in 55 and $49 \%$ yield respectively, representing cumulative yields of $\geq 70 \%$ for each ring closure. 


\section{Conclusions}

This article demonstrates the necessity for converting 4,6-di(cycloamino)-1,3-phenylenediamines to dianilide/diacetamide prior to imidazobenzimidazole formation. New evidence is presented for nitroso and $N$ oxide intermediates in the respective oxidative cyclizations of 2-(cycloamino)anilines and 2(cycloamino)anilides. Oxetane is incorporated into ring-fused imidazobenzimidazoles for the first time, with Oxone in acetic acid allowing the formation of both $[4,5-f]$ and $[5,4-f]$ isomers. Future work should complete overall oxidation of the aromatic part of $\mathbf{4}$ and $\mathbf{5}$ to give the (imino)quinone NQO1 substrates.

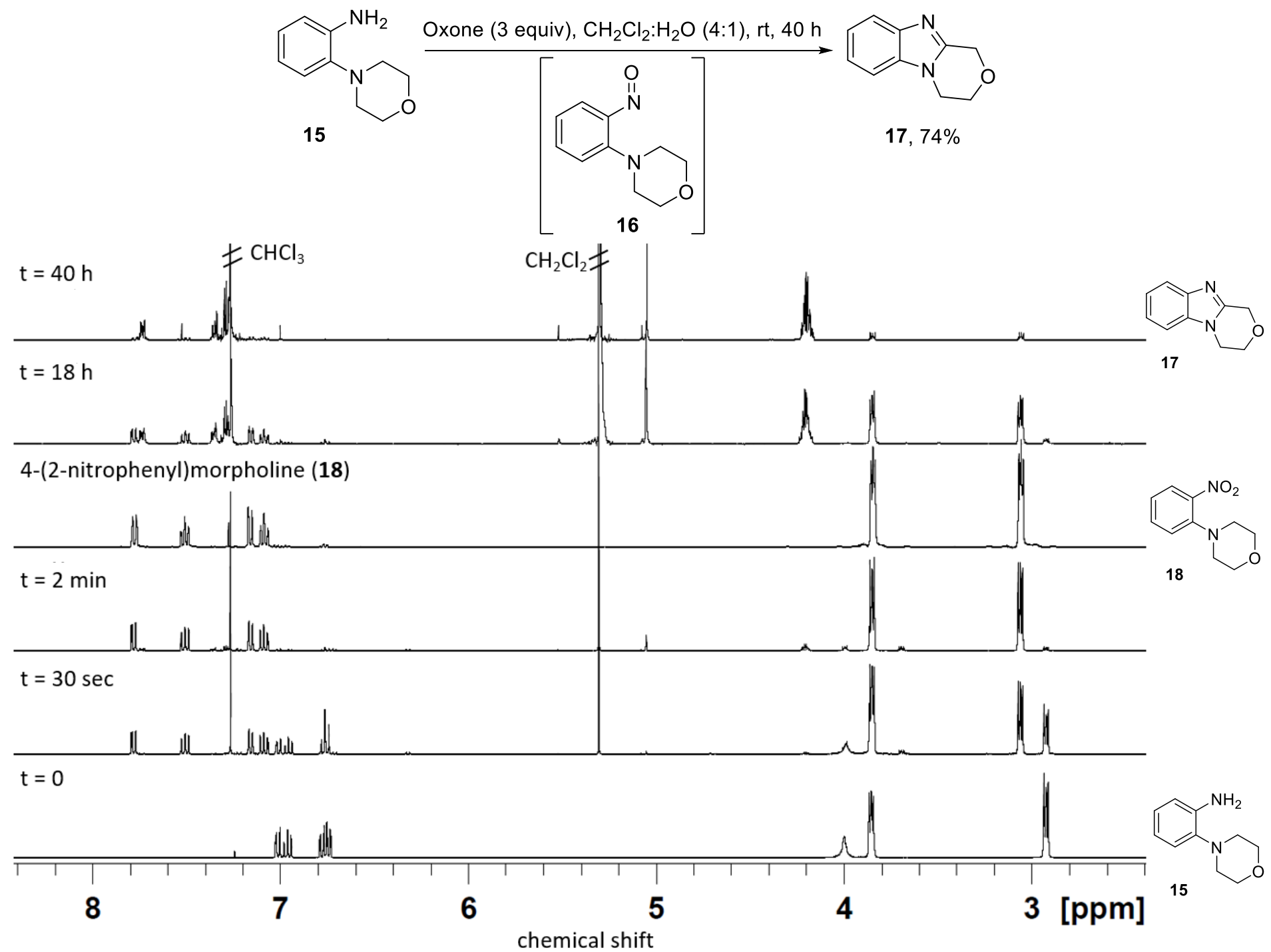

Scheme 5. ${ }^{1} \mathrm{H}$ NMR reaction monitoring, providing evidence for the $o$-nitroso-tert-aniline intermediate 16.

\section{Experimental Section}

Materials. Pd-C (Sigma Aldrich, 5\% wt. loading), EtOAc (VWR, 99.9\%), pet. ether (Fisher Scientific, 40-60 ${ }^{\circ} \mathrm{C}$, Extra Pure, SLR), pivaloyl chloride (Sigma Aldrich, 99\%), Oxone (Sigma Aldrich, $\mathrm{KHSO}_{5} \cdot 0.5 \mathrm{KHSO}_{4} \cdot 0.5 \mathrm{~K}_{2} \mathrm{SO}_{4}$ ), HFIP (Fluorochem, 99\%), $\mathrm{NaHCO}_{3}$ (Fisher Scientific, $\geq 99.7 \%$ ), 1,5-difluoro-2,4-dinitrobenzene (Sigma Aldrich, 
97\%), MeCN (Fisher Scientific, HPLC grade), piperidine (ACROS Organics ${ }^{\mathrm{TM}}$, 99\%), AcOH (Fisher Scientific, glacial), $\mathrm{Ac}_{2} \mathrm{O}$ (ACROS Organics $\left.{ }^{\mathrm{TM}}, 99+\%\right), \mathrm{MsOH}$ (Fluorochem, >98\%), $\mathrm{Na}_{2} \mathrm{CO}_{3}$ (Fisher Scientific, 99.5\%), $\mathrm{D}_{2} \mathrm{O}$ (Fluorochem, >99.9\%), and $\mathrm{MgSO}_{4}$ (Fisher Scientific, Extra Pure, SLR, Dried) were used as received. $\mathrm{CH}_{2} \mathrm{Cl}_{2}$ (Fisher Scientific, 99.8\%) was distilled over $\mathrm{CaH}_{2}$ (ACROS Organics ${ }^{\mathrm{TM}}$, ca. 93\%, extra pure, 0-2 mm grain size) prior to use. 1,1'-(4,6-Dinitro-1,3-phenylene)dipiperidine was prepared (1.198 $\mathrm{g}, 92 \%)$ by modifying the reported procedure, ${ }^{2}$ by reacting piperidine $(2.50 \mathrm{~mL}, 29.00 \mathrm{mmol})$ and $\mathrm{NaHCO}_{3}(1.600 \mathrm{~g}, 19.50 \mathrm{mmol})$ with 1,5-difluoro-2,4-dinitrobenzene $(0.800 \mathrm{~g}, 3.90 \mathrm{mmol})$ in THF $(30 \mathrm{~mL})$ at room temperature for $1 \mathrm{~h}$. The synthesis of 4-(2-nitrophenyl)morpholine (18) (1.042 g, 83\%) was achieved by $\mathrm{S}_{N} A r$ of morpholine (1.56 ml, $18.09 \mathrm{mmol}$, Alfa Aesar, 99\%) onto 1-fluoro-2-nitrobenzene (0.850 g, $6.03 \mathrm{mmol}$, Fluorochem, 99\%) in the absence of solvent at $110{ }^{\circ} \mathrm{C}$ for $1 \mathrm{~h}$. 2-(Morpholin-4-yl)aniline (15) $)^{13}$ was obtained in 91\% yield, through reduction of 18 with iron powder, according to our previously reported method. ${ }^{9,10,12,14}$ The Aldabbagh group has previously described the synthesis of bis(2-oxa-7-azaspiro[3.5]nonan-7-ium) ethanedioate (oxalate salt of 1), ${ }^{10}$ and 1,4-difluoro-2,5-dinitrobenzene. ${ }^{4}$ All reactions (apart from those using aqueous solutions) were carried out under an inert nitrogen atmosphere. Thin Layer Chromatography (TLC) was carried out on TLC silica gel $60 \mathrm{~F}_{254}$ plates, and preparative TLC was done on TLC Silica Gel $60 \mathrm{~F}_{254}$ glass plates. Flash column chromatography was carried out on silica gel (Apollo Scientific 60/40-63 $\mu \mathrm{m}$ ).

(a)

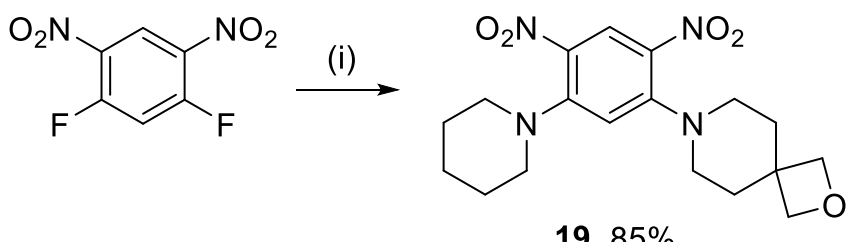

$19,85 \%$

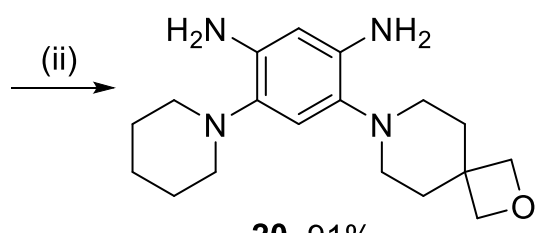

20, $91 \%$

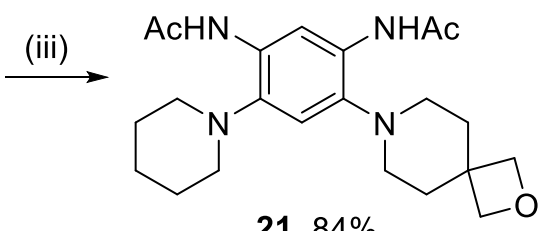

21, $84 \%$

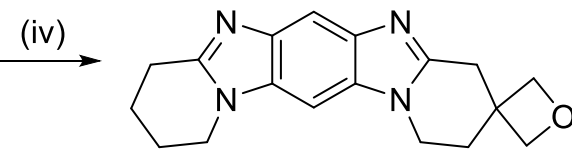

4, $55 \%$

(b)

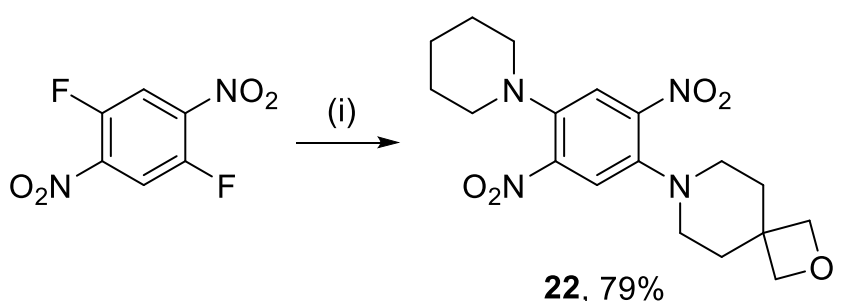

22, $79 \%$
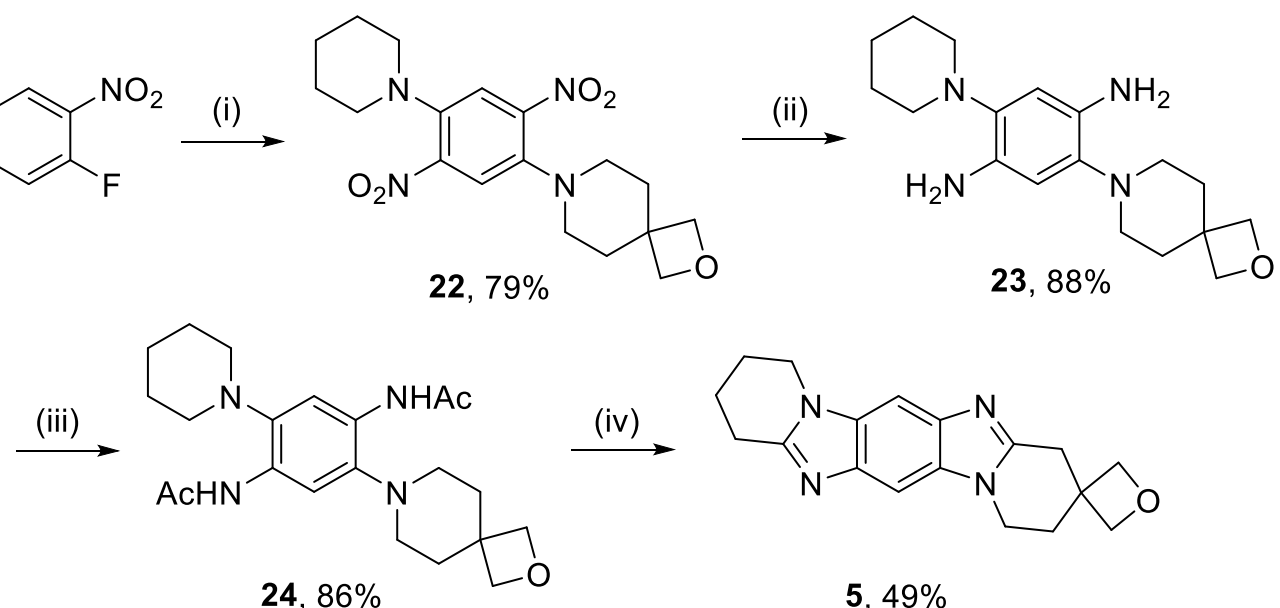

5, $49 \%$

Scheme 6. Synthesis of (a) imidazo[4,5-f]benzimidazole 4 and (b) imidazo[5,4-f]benzimidazole 5: (i) 1 oxalate salt, $\mathrm{NaHCO}_{3}, \mathrm{MeCN}$ (aq), rt, 16 h; piperidine, rt, 1 h; (ii) $\mathrm{H}_{2}$ (balloon), Pd-C, EtOAc, rt, 18 h; (iii) Ac $\mathrm{Ac}_{2}$ (10 equiv), $\mathrm{AcOH}, 80^{\circ} \mathrm{C}, 30 \mathrm{~min}$; (iv) Oxone ( 6 equiv), $\mathrm{AcOH}, 40^{\circ} \mathrm{C}, 7 \mathrm{~h}$. 
Instruments. Melting points were measured on a Stuart Scientific melting point apparatus SMP1. IR spectra were recorded using a PerkinElmer Spec 1 with ATR attached. NMR spectra were recorded using a Bruker Avance II $400 \mathrm{MHz}$ spectrometer. Chemical shifts are in ppm, relative to $\mathrm{Me}_{4} \mathrm{Si}^{1}{ }^{1} \mathrm{H} \mathrm{NMR} \mathrm{NH}$ amide and amine assignments were verified by $\mathrm{D}_{2} \mathrm{O}$ exchange experiments. ${ }^{13} \mathrm{C} \mathrm{NMR}$ spectra are at $100 \mathrm{MHz}$ with complete proton decoupling and assignments supported by Distortionless Enhancement by Polarization Transfer (DEPT). NMR assignments for synthetic targets 4 and 5 used data of reported spirocyclic oxetane ring-fused compounds. ${ }^{9,10}$ HRMS spectra of compounds 4, 5, 19, 21, 22 and 24 were obtained at the National University of Ireland Galway, using an ESI time-of-flight mass spectrometer (TOFMS) on a Waters LCT Mass Spectrometry instrument. HRMS spectra of all other compounds were obtained at the National Mass Spectrometry Facility at Swansea University using a Waters Xevo G2-S mass spectrometer with an Atmospheric Solids Analysis Probe (ASAP) or Thermo Scientific LTQ Orbitrap XL instrument with Nanospray Ionization (NSI). The precision of all accurate mass measurements was better than $5 \mathrm{ppm}$.

Synthesis of 4,6-di(piperidin-1-yl)benzene-1,3-diamine (6). 1,1'-(4,6-Dinitro-1,3-phenylene)dipiperidine $(0.750 \mathrm{~g}, 2.25 \mathrm{mmol})$, and $\mathrm{Pd}-\mathrm{C}(75 \mathrm{mg})$ in EtOAc $(50 \mathrm{~mL})$ were stirred under $\mathrm{H}_{2}$ at room temperature for $24 \mathrm{~h}$. The mixture was filtered through Celite and evaporated to dryness. The residue was purified by column chromatography using gradient elution of pet. ether and EtOAc to give the title compound $(0.512 \mathrm{~g}, 83 \%)$ as a brown solid; $R_{\mathrm{f}} 0.32$ (2:1 pet. ether:EtOAc); $\mathrm{mp} 172-174{ }^{\circ} \mathrm{C} ; \mathrm{v}_{\max }$ (neat, $\mathrm{cm}^{-1}$ ) 3378, 3264, 2946, 2923, 2844, 2799, 2742, 1626, 1518, 1466, 1441, 1382, 1339, 1296, 1273, 1257, 1242, 1214, 1201, 1149, 1129, 1111, 1037, 1029; ${ }^{1} \mathrm{H}$ NMR $\left(400 \mathrm{MHz}, \mathrm{CDCl}_{3}\right) \delta: 6.69$ (s, $\left.1 \mathrm{H}\right), 6.11$ (s, 1H), 4.00-3.31 (br.s, 4H, $\mathrm{NH}_{2}$ ), 2.85-2.55 (br.s, 8H), 1.64-1.55 (m, 8H), 1.54-1.36 (br.s, 4H); ${ }^{13} \mathrm{C} \mathrm{NMR} \mathrm{(100} \mathrm{MHz,} \mathrm{CDCl}_{3}$ ) $\delta: 138.6,132.5$ (both C), 112.6, 102.1 (both $\mathrm{CH}$ ), 53.6, 27.1, 24.4 (all $\mathrm{CH}_{2}$ ); HRMS (ASAP) $\mathrm{m} / \mathrm{z}[\mathrm{M}+\mathrm{H}]^{+}$found 275.2234, $\mathrm{C}_{16} \mathrm{H}_{27} \mathrm{~N}_{4}$ requires 275.2236.

Anilide formation. Pivaloyl chloride $(45 \mu \mathrm{L}, 0.37 \mathrm{mmol})$ in $\mathrm{CH}_{2} \mathrm{Cl}_{2}(8 \mathrm{~mL})$ was added via syringe pump at a rate of $2 \mathrm{~mL} / \mathrm{h}$ to diamine $6(0.103 \mathrm{~g}, 0.37 \mathrm{mmol})$ in $\mathrm{CH}_{2} \mathrm{Cl}_{2}(8 \mathrm{~mL})$. The mixture was stirred for a further $2 \mathrm{~h}$ at room temperature. $\mathrm{H}_{2} \mathrm{O}(20 \mathrm{~mL})$ was added, and the mixture was extracted with $\mathrm{CH}_{2} \mathrm{Cl}_{2}(2 \times 20 \mathrm{~mL})$. The combined organic extracts were dried $\left(\mathrm{MgSO}_{4}\right)$, evaporated and purified by column chromatography using gradient elution of pet. ether and EtOAc.

$N$-[5-Amino-2,4-di(piperidin-1-yl)phenyl]-2,2-dimethylpropanamide (10). (81 mg, 61\%); pale brown solid; $R_{\mathrm{f}}$ 0.44 (7:3 pet. ether:EtOAc); $\mathrm{mp} 149-151^{\circ} \mathrm{C} ; \mathrm{v}_{\max }$ (neat, $\mathrm{cm}^{-1}$ ) 3423, 3333, 2931, 2851, 2801, 2739, 2360, 1669 (C=O), 1618, 1593, 1519, 1480, 1435, 1377, 1364, 1321, 1272, 1235, 1206, 1193, 1150, 1121, 1110, 1063,

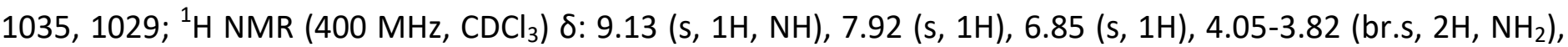
2.90-2.62 (m, 8H), 1.75-1.64 (m, 8H), 1.63-1.47 (br.s, 4H), $\left.1.31(\mathrm{~s}, 9 \mathrm{H}, \mathrm{Me}) ;{ }^{13} \mathrm{C} \mathrm{NMR} \mathrm{(100} \mathrm{MHz,} \mathrm{CDCl}_{3}\right) \delta: 176.2$ (C=O), 138.9, 135.7, 133.8, 130.5 (all C), 112.4, 105.5 (both $\mathrm{CH}$ ), 54.3, 53.0 (both $\mathrm{CH}_{2}$ ), 40.0 (C), 27.8 (Me), 27.3, 26.9, 24.4, 24.1 (all $\mathrm{CH}_{2}$ ); HRMS (ASAP) $\mathrm{m} / \mathrm{z}[\mathrm{M}+\mathrm{H}]^{+}$found 359.2809, $\mathrm{C}_{21} \mathrm{H}_{35} \mathrm{~N}_{4} \mathrm{O}$ requires 359.2811.

$N, N^{\prime}$-[4,6-Di(piperidin-1-yl)-1,3-phenylene]bis(2,2-dimethylpropanamide) (11). (22 mg, 14\%); brown solid; $R_{\mathrm{f}}$ 0.37 (pet. ether:EtOAc); $m p \quad 236-237^{\circ} \mathrm{C} ; v_{\max }$ (neat, $\mathrm{cm}^{-1}$ ) 3344, 2949, 2930, 2917, 2849, 1682 (C=0), 1596, $1519,1480,1448,1428,1393,1377,1362,1356,1340,1309,1269,1219,1190,1162,1150,1103,1063,1027$; ${ }^{1} \mathrm{H}$ NMR $\left(400 \mathrm{MHz}_{\mathrm{CDCl}}\right)$ ) $8: 9.36(\mathrm{~s}, 1 \mathrm{H}), 8.77(\mathrm{~s}, 1 \mathrm{H}, \mathrm{NH}), 6.95(\mathrm{~s}, 1 \mathrm{H}), 2.74(\mathrm{t}, J 5.2 \mathrm{~Hz}, 8 \mathrm{H}), 1.75-1.68(\mathrm{~m}, 8 \mathrm{H})$, 1.62-1.55 (m, 4H), $\left.1.30(\mathrm{~s}, 18 \mathrm{H}, \mathrm{Me}) ;{ }^{13} \mathrm{C} \mathrm{NMR} \mathrm{(100} \mathrm{MHz,} \mathrm{CDCl}_{3}\right)$ $\delta: 175.6(\mathrm{C}=0), 138.0,130.8$ (both C), 112.4, 110.7 (both $\mathrm{CH}$ ), $53.9\left(\mathrm{CH}_{2}\right), 39.9$ (C), 27.8 (Me), 27.1, $24.1\left(\mathrm{CH}_{2}\right) ; \mathrm{HRMS}$ (ASAP) $\mathrm{m} / z$ [M+H] $]^{+}$found 443.3384, $\mathrm{C}_{26} \mathrm{H}_{43} \mathrm{~N}_{4} \mathrm{O}_{2}$ requires 443.3386 .

Reaction of amine-anilide 10 with Oxone (in the absence of acid). Oxone $(0.277 \mathrm{~g}, 0.90 \mathrm{mmol})$ was added to amine-anilide 10 (65 mg, $0.18 \mathrm{mmol}$ ) in HFIP (3.6 mL, 10\% aq.) and stirred at room temperature for $16 \mathrm{~h}$. $\mathrm{H}_{2} \mathrm{O}$ $(10 \mathrm{~mL})$ was added, and the mixture extracted with $\mathrm{CH}_{2} \mathrm{Cl}_{2}(2 \times 10 \mathrm{~mL})$. The organic extracts were dried $\left(\mathrm{MgSO}_{4}\right)$, evaporated and purified by column chromatography using gradient elution of $\mathrm{CH}_{2} \mathrm{Cl}_{2}$ and $\mathrm{MeOH}$. 


\section{2,2-Dimethyl-N-[8-(1-oxidopiperidin-1-yl)-1,2,3,4-tetrahydropyrido[1,2-a]benzimidazol-7-yl]propenamide}

(12). (39 mg, 58\%); pale brown solid; $R_{\mathrm{f}} 0.26\left(9: 1 \mathrm{CH}_{2} \mathrm{Cl}_{2}: \mathrm{MeOH}\right) ; \mathrm{mp}$ (decomp. $\left.>161{ }^{\circ} \mathrm{C}\right) ; v_{\max }\left(\mathrm{neat}, \mathrm{cm}^{-1}\right) 2952$, 2927, 2866, 1652 (C=O), 1594, 1497, 1474, 1430, 1417, 1366, 1327, 1273, 1199, 1148, 1011; ${ }^{1} \mathrm{H}$ NMR (400

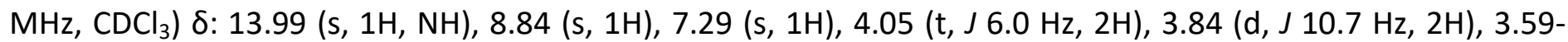
$3.47(\mathrm{~m}, 2 \mathrm{H}), 3.08(\mathrm{t}, J 6.3 \mathrm{~Hz}, 2 \mathrm{H}), 2.80-2.70(\mathrm{~m}, 2 \mathrm{H}), 2.17-2.11(\mathrm{~m}, 2 \mathrm{H}), 2.05-2.01(\mathrm{~m}, 2 \mathrm{H}), 1.89(\mathrm{~d}, J 13.0 \mathrm{~Hz}$, $1 \mathrm{H}), 1.75(\mathrm{~d}, J 13.6 \mathrm{~Hz}, 2 \mathrm{H}), 1.48-1.37(\mathrm{~m}, 1 \mathrm{H}), 1.35(\mathrm{~s}, 9 \mathrm{H}, \mathrm{Me}) ;{ }^{13} \mathrm{C} \mathrm{NMR}\left(100 \mathrm{MHz}, \mathrm{CDCl}_{3}\right) \delta: 176.9(\mathrm{C}=\mathrm{O})$, 154.1, 142.7, 137.6, 130.6, 129.1 (all C), 114.0, 98.2 (both $\mathrm{CH}$ ), 66.5, 42.5 (both $\mathrm{CH}_{2}$ ), 40.0 (C), 27.8 (Me), 25.5, 22.5, 21.9, 20.9, 20.5 (all $\mathrm{CH}_{2}$ ); HRMS (ASAP) $\mathrm{m} / \mathrm{z}[\mathrm{M}+\mathrm{H}]^{+}$found 371.2447, $\mathrm{C}_{21} \mathrm{H}_{31} \mathrm{~N}_{4} \mathrm{O}_{2}$ requires $371.2447, \mathrm{~m} / \mathrm{z}$ 372 (25\%), 371 (M+1, 100\%), 356 (16\%), 355 (M-16, 73\%), 354 (26\%), 353 (M-18, 97\%), 269 (M-102, 28\%).

2,2-Dimethyl-N-[8-(piperidin-1-yl)-1,2,3,4-tetrahydropyrido[1,2-a]benzimidazol-7-yl]propenamide (13). (19 $\mathrm{mg}, 29 \%) ;$ brown solid; $R_{\mathrm{f}} 0.44$ (9:1 $\left.\mathrm{CH}_{2} \mathrm{Cl}_{2}: \mathrm{MeOH}\right) ; \mathrm{mp} 171-173{ }^{\circ} \mathrm{C} ; \mathrm{v}_{\max }$ (neat, $\mathrm{cm}^{-1}$ ) 3340, 2935, 2863, 2809, 1667 (C=O), 1589, 1511, 1473, 1440, 1418, 1365, 1322, 1268, 1242, 1194, 1146, 1136, 1064, 1034; ${ }^{1} \mathrm{H}$ NMR $\left(400 \mathrm{MHz}_{\mathrm{CDCl}}\right)$ ) $: 9.25(\mathrm{~s}, 1 \mathrm{H}, \mathrm{NH}), 8.74(\mathrm{~s}, 1 \mathrm{H}), 7.08(\mathrm{~s}, 1 \mathrm{H}), 4.03(\mathrm{t}, J 6.1 \mathrm{~Hz}, 2 \mathrm{H}), 3.05(\mathrm{t}, J 6.4 \mathrm{~Hz}, 2 \mathrm{H}), 2.96-$ 2.67 (br.s, 4H), 2.16-2.06 (m, 2H), 2.05-1.95 (m, 2H), 1.84-1.54 (m, 6H), $1.36(\mathrm{~s}, 9 \mathrm{H}, \mathrm{Me}) ;{ }^{13} \mathrm{C}\left\{{ }^{1} \mathrm{H}\right\} \mathrm{NMR}(100$

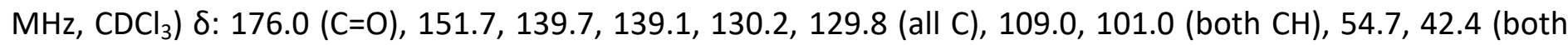
$\mathrm{CH}_{2}$ ), 40.1 (C), 27.9 (Me), 27.2, 25.3, 24.1, 22.7, 20.7 (all $\mathrm{CH}_{2}$ ); HRMS (ASAP) $\mathrm{m} / \mathrm{z}[\mathrm{M}+\mathrm{H}]^{+}$found 355.2495, $\mathrm{C}_{21} \mathrm{H}_{31} \mathrm{~N}_{4} \mathrm{O}$ requires 355.2498 .

Ring-closure with acid. $\mathrm{MsOH}(0.05 \mathrm{~mL}, 0.77 \mathrm{mmol})$ was added to a solution of $N$-oxide $12(28 \mathrm{mg}, 0.08 \mathrm{mmol})$ in $\mathrm{CH}_{2} \mathrm{Cl}_{2}\left(0.8 \mathrm{~mL}\right.$ ) and stirred at room temperature for $5 \mathrm{~h}$. $\mathrm{Na}_{2} \mathrm{CO}_{3}$ (satd., $2 \mathrm{~mL}$ ) was added, and the mixture extracted with $\mathrm{CH}_{2} \mathrm{Cl}_{2}(3 \times 2 \mathrm{~mL})$. The organic extracts were dried $\left(\mathrm{MgSO}_{4}\right)$, evaporated and purified by preparative TLC.

2,2-Dimethyl-N-[8-(piperidin-1-yl)-1,2,3,4-tetrahydropyrido[1,2-a]benzimidazol-7-yl]propenamide (13). (12 $\mathrm{mg}, 44 \%) ; R_{\mathrm{f}} 0.44\left(9: 1 \mathrm{CH}_{2} \mathrm{Cl}_{2}: \mathrm{MeOH}\right)$; spectral data and melting point consistent with the above.

1,2,3,4,8,9,10,11-Octahydropyrido[1,2-a]pyrido[1',2':1,2]imidazo[4,5-f]benzimidazole (14).,16 (8 mg, 42\%); off-white solid; $R_{\mathrm{f}} 0.20$ (9:1 $\mathrm{CH}_{2} \mathrm{Cl}_{2}: \mathrm{MeOH}$ ); mp (decomp. $>261{ }^{\circ} \mathrm{C}$; lit. m.p. ${ }^{3}$ decomp. $\left.266-270{ }^{\circ} \mathrm{C}\right) ;{ }^{1} \mathrm{H} \mathrm{NMR}(400$ $\left.\mathrm{MHz}_{,} \mathrm{CDCl}_{3}\right) \delta: 7.95(\mathrm{~d}, J 0.8 \mathrm{~Hz}, 1 \mathrm{H}), 7.04(\mathrm{~d}, J 0.8 \mathrm{~Hz}, 1 \mathrm{H}), 4.09(\mathrm{t}, J 6.1 \mathrm{~Hz}, 4 \mathrm{H}), 3.11(\mathrm{t}, J 6.4 \mathrm{~Hz}, 4 \mathrm{H}), 2.20-2.13$ $(\mathrm{m}, 4 \mathrm{H}), 2.07-2.00(\mathrm{~m}, 4 \mathrm{H})$.

Reaction of aniline 15 with Oxone. Aniline 15 (0.356 g, $2.00 \mathrm{mmol})$ and Oxone $(1.846 \mathrm{~g}, 6.01 \mathrm{mmol})$ in $\mathrm{CH}_{2} \mathrm{Cl}_{2}$ $(4 \mathrm{~mL})$ and $\mathrm{H}_{2} \mathrm{O}(1 \mathrm{~mL})$ were rapidly stirred at rt. Aliquots $(0.2 \mathrm{~mL})$ were taken at the times shown in Scheme 5, quenched with water $(0.5 \mathrm{~mL})$, and extracted with $\mathrm{CDCl}_{3}(0.5 \mathrm{~mL})$ for ${ }^{1} \mathrm{H} \mathrm{NMR}$ analysis. After $2 \mathrm{~min}$, nitrobenzene 18 was the apparent major product; ${ }^{1} \mathrm{H} \mathrm{NMR}\left(400 \mathrm{MHz}, \mathrm{CDCl}_{3}\right) \delta: 7.78(\mathrm{dd}, J 8.1 \mathrm{~Hz}, 1.6 \mathrm{~Hz}, 1 \mathrm{H})$, 7.53-7.48 (m, 1H), 7.16 (dd, J 8.3, 1.2 Hz, 1H), 7.11-7.06 (m, 1H), 3.85 (t, J 4.6 Hz, 4H), 3.06 (t, J 4.6 Hz, 4H). The reaction under the same conditions was stirred for $40 \mathrm{~h} . \mathrm{H}_{2} \mathrm{O}(5 \mathrm{~mL})$ was added, and the mixture extracted with $\mathrm{CH}_{2} \mathrm{Cl}_{2}(2 \times 6 \mathrm{~mL})$. The organic extracts were dried $\left(\mathrm{MgSO}_{4}\right)$, evaporated and purified by column chromatography using gradient elution of pet. ether and EtOAc to give 3,4-dihydro-1H-[1,4]oxazino[4,3a]benzimidazole (17) $(0.259 \mathrm{~g}, 74 \%)$ as a pale brown solid; $R_{\mathrm{f}} 0.21$ (EtOAC); mp $123-125{ }^{\circ} \mathrm{C}$ (lit m.p. ${ }^{17} 129-130$ $\left.{ }^{\circ} \mathrm{C}\right) ;{ }^{1} \mathrm{H}$ NMR $\left(400 \mathrm{MHz}, \mathrm{CDCl}_{3}\right)$ 8: 7.76-7.72 (m, 1H), 7.37-7.32 (m, 1H), 7.31-7.27 (m, 2H), 5.05 (s, 2H), 4.24-4.16 $(\mathrm{m}, 4 \mathrm{H})$. Spectral data and melting point were consistent with reported. ${ }^{20}$

Procedure for the synthesis of nitrobenzenes 19 and 22. Oxalate salt of $1(0.540 \mathrm{~g}, 1.57 \mathrm{mmol}), \mathrm{NaHCO}_{3}$ (1.020 g, $12.48 \mathrm{mmol})$ and 1,5-difluoro-2,4-dinitrobenzene or 1,4-difluoro-2,5-dinitrobenzene (0.636 g, 3.12 $\mathrm{mmol})$ in $\mathrm{MeCN}(40 \mathrm{ml})$ and $\mathrm{H}_{2} \mathrm{O}(4 \mathrm{ml})$ were stirred at room temperature for $16 \mathrm{~h}$. Piperidine $(3.00 \mathrm{ml}, 30.42$ $\mathrm{mmol}$ ) was added to the mixture and stirred for a further $1 \mathrm{~h}$. The mixture was evaporated, EtOAc (50 $\mathrm{mL})$ added, washed with brine $(3 \times 50 \mathrm{~mL})$, dried $\left(\mathrm{MgSO}_{4}\right)$, and evaporated to dryness. The residue was purified by column chromatography using gradient elution of pet. ether and EtOAc. 
1-(2,4-Dinitro-5-piperidin-1-ylphenyl)-2-oxa-7-azaspiro[3.5]nonane (19). (0.993 g, 85\%); yellow solid; $R_{\mathrm{f}} 0.32$ (1:1 pet. Ether:EtOAc); $m p 156-157^{\circ} \mathrm{C} ; v_{\max }$ (neat, $\left.\mathrm{cm}^{-1}\right) 2941,2855,1600,1555,1489\left(\mathrm{NO}_{2}\right), 1441,1326\left(\mathrm{NO}_{2}\right)$, 1299, 1254; ${ }^{1} \mathrm{H}$ NMR $\left(400 \mathrm{MHz}, \mathrm{CDCl}_{3}\right) \delta: 8.66(\mathrm{~s}, 1 \mathrm{H}), 6.25(\mathrm{~s}, 1 \mathrm{H}), 4.48\left(\mathrm{~s}, 4 \mathrm{H}, \mathrm{OCH}_{2}\right), 3.12(\mathrm{t}, J 5.3 \mathrm{~Hz}, 4 \mathrm{H}), 3.04$ (t, J $5.5 \mathrm{~Hz}, 4 \mathrm{H}), 2.06(\mathrm{t}, J 5.5 \mathrm{~Hz}, 4 \mathrm{H}), 1.76-1.69(\mathrm{~m}, 4 \mathrm{H}), 1.69-1.62(\mathrm{~m}, 2 \mathrm{H}) ;{ }^{13} \mathrm{C} \mathrm{NMR}(100 \mathrm{MHz}, \mathrm{CDCl})_{3} \delta: 150.8$, 131.1, 130.6 (all C), 130.0, 107.4 (both $\mathrm{CH}$ ), $81.4\left(\mathrm{OCH}_{2}\right), 52.3,48.6$ (both $\left.\mathrm{CH}_{2}\right), 38.5(\mathrm{C}), 34.4,25.5,23.9$ (all $\left.\mathrm{CH}_{2}\right) ; \mathrm{HRMS}(\mathrm{ESI}) \mathrm{m} / \mathrm{z}[\mathrm{M}+\mathrm{H}]^{+}$found $377.1813, \mathrm{C}_{18} \mathrm{H}_{25} \mathrm{~N}_{4} \mathrm{O}_{5}$ requires 377.1825 .

1-(2,5-Dinitro-4-piperidin-1-ylphenyl)-2-oxa-7-azaspiro[3.5]nonane (22). (0.926 g, 79\%); red-brown solid; $R_{\mathrm{f}}$ 0.49 (4:1 pet. Ether:EtOAc); $m p 148-149^{\circ} \mathrm{C} ; v_{\max }\left(\right.$ neat, $\left.\mathrm{cm}^{-1}\right)$ 2929, 2859, 2810, 1533, $1495\left(\mathrm{NO}_{2}\right), 1466,1445$, $1410,1385,1346\left(\mathrm{NO}_{2}\right), 1324\left(\mathrm{NO}_{2}\right), 1272,1236,1225,1209,1131,1042 ;{ }^{1} \mathrm{H} \mathrm{NMR}(400 \mathrm{MHz}, \mathrm{CDCl}) \delta: 7.45(\mathrm{~s}$, $1 \mathrm{H}), 7.41(\mathrm{~s}, 1 \mathrm{H}), 4.45\left(\mathrm{~s}, 4 \mathrm{H}, \mathrm{OCH}_{2}\right), 2.95(\mathrm{t}, J 5.3 \mathrm{~Hz}, 4 \mathrm{H}), 2.87(\mathrm{t}, J 5.5 \mathrm{~Hz}, 4 \mathrm{H}), 1.99$ (t, J $\left.5.5 \mathrm{~Hz}, 4 \mathrm{H}\right), 1.71-1.65$

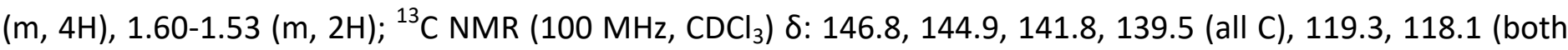
$\mathrm{CH}$ ), $81.6\left(\mathrm{OCH}_{2}\right), 53.1,49.8$ (both $\left.\mathrm{CH}_{2}\right), 38.2(\mathrm{C}), 34.9,25.9,23.8$ (all $\left.\mathrm{CH}_{2}\right) ; \mathrm{HRMS}$ (ESI) $\mathrm{m} / z[\mathrm{M}+\mathrm{H}]^{+}$found $377.1818, \mathrm{C}_{18} \mathrm{H}_{25} \mathrm{~N}_{4} \mathrm{O}_{5}$ requires 377.1825 .

Procedure for the synthesis of diamines 20 and 23. Dinitrobenzene 19 or $22(0.500 \mathrm{~g}, 1.33 \mathrm{mmol})$, and Pd-C $(50 \mathrm{mg})$ in EtOAc $(50 \mathrm{~mL})$ were stirred under $\mathrm{H}_{2}$ (balloon) at room temperature for $16 \mathrm{~h}$. The mixture was filtered through Celite and evaporated to dryness.

4-(2-Oxa-7-azaspiro[3.5]nonan-7-yl)-6-(piperidin-1-yl)benzene-1,3-diamine (20). (0.381 g, 91\%); brown solid; $\mathrm{mp} 187-188^{\circ} \mathrm{C} ; v_{\max }\left(\right.$ neat, $\mathrm{cm}^{-1}$ ) 3372, 3264, 2951, 2920, 2848, 2740, 1626, 1519, 1468, 1442, 1380, 1296, $1278,1257,1246,1215,1149,1132,1113,1036,1026 ;{ }^{1} \mathrm{H} \mathrm{NMR}\left(400 \mathrm{MHz}, \mathrm{CDCl}_{3}\right) \delta: 6.60(\mathrm{~s}, 1 \mathrm{H}), 6.07(\mathrm{~s}, 1 \mathrm{H})$, 4.40 (s, 4H, OCH ), 3.78-3.64 (br.s, 4H, NH ), 2.81-2.41 (br.s, 8H), 2.02-1.79 (br.s, 4H), 1.64-1.53 (m, $4 \mathrm{H}), 1.52-$ 1.36 (br.s, 2H); ${ }^{13} \mathrm{C} \mathrm{NMR} \mathrm{(100} \mathrm{MHz,} \mathrm{CDCl}_{3}$ ) $\delta: 138.9,138.5,132.5,131.2$ (all C), 112.4, 102.1 (both CH), 82.1 $\left(\mathrm{OCH}_{2}\right.$ ), 53.6, 49.6 (both $\mathrm{CH}_{2}$ ), 38.5 (C), 36.1, 27.1, 24.4 (all $\mathrm{CH}_{2}$ ); HRMS (NSI) $\mathrm{m} / z[\mathrm{M}+\mathrm{H}]^{+}$found 317.2342, $\mathrm{C}_{18} \mathrm{H}_{29} \mathrm{~N}_{4} \mathrm{O}_{1}$ requires 317.2336 .

2-(2-Oxa-7-azaspiro[3.5]nonan-7-yl)-5-(piperidin-1-yl)benzene-1,4-diamine (23). (0.369 g, 88\%); grey solid; $\mathrm{mp} 197-199^{\circ} \mathrm{C} ; v_{\max }\left(\right.$ neat, $\mathrm{cm}^{-1}$ ) 3386, 3302, 3273, 3162, 2924, 2855, 2800, 2739, 1585, 1511, 1462, 1450, $1432,1342,1301,1246,1200,1111,1033 ;{ }^{1} \mathrm{H} \mathrm{NMR}\left(400 \mathrm{MHz}, \mathrm{CDCl}_{3}\right) \delta: 6.40(\mathrm{~s}, 1 \mathrm{H}), 6.35(\mathrm{~s}, 1 \mathrm{H}), 4.40(\mathrm{~s}, 4 \mathrm{H}$, $\mathrm{OCH}_{2}$ ), 3.64-3.40 (br.s, 4H, NH $\mathrm{N}_{2}$ ), 2.78-2.54 (m, 8H), 1.96-1.84 (br.s, 4H), 1.65-1.56 (m, 4H), 1.53-1.40 (br.s, 2H); ${ }^{13} \mathrm{C}\left\{{ }^{1} \mathrm{H}\right\}$ NMR $\left(100 \mathrm{MHz}, \mathrm{CDCl}_{3}\right) \delta: 137.7,136.1,133.5,133.3$ (all C), 108.1, 107.8 (both CH), 82.0 $\left(\mathrm{OCH}_{2}\right), 53.0$, 49.0 (both $\mathrm{CH}_{2}$ ), 38.5 (C), 35.9, 26.9, 24.4 (all $\mathrm{CH}_{2}$ ); HRMS (NSI) $\mathrm{m} / z[\mathrm{M}+\mathrm{H}]^{+}$found 317.2339, $\mathrm{C}_{18} \mathrm{H}_{29} \mathrm{~N}_{4} \mathrm{O}_{1}$ requires 317.2336 .

Procedure for the synthesis of diacetamides $\mathbf{2 1}$ and 24. Diamine $\mathbf{2 0}$ or $\mathbf{2 3}$ (0.354 g, $1.12 \mathrm{mmol})$ in $\mathrm{Ac}_{2} \mathrm{O}$ (1.06 $\mathrm{mL}, 11.20 \mathrm{mmol})$ and $\mathrm{AcOH}(30 \mathrm{~mL})$ was stirred at $80{ }^{\circ} \mathrm{C}$ for $30 \mathrm{~min}$. The mixture was evaporated, $\mathrm{NaHCO}_{3}(5 \%$, $100 \mathrm{~mL}$ ) added and stirred for $1 \mathrm{~h}$. The precipitate was collected, washed with water, dried, and recrystallized from EtOAc.

$\boldsymbol{N}, \boldsymbol{N}^{\prime}$-[4-(2-Oxa-7-azaspiro[3.5]nonan-7-yl)-6-piperidin-1-yl-1,3-phenylene]diacetamide (21). (0.375 g, 84\%); white solid; $\mathrm{mp} 226-228{ }^{\circ} \mathrm{C} ; \mathrm{v}_{\max }\left(\right.$ neat, $\mathrm{cm}^{-1}$ ) 3291, 2935, 2856, 2797, 2735, 1728, 1672 (C=O), 1659, 1589, $1524,1491,1420,1378,1368,1289,1276,1256,1232,1218,1196,1158,1136,1122,1112,1066,1032 ;{ }^{1} \mathrm{H}$ NMR $\left(400 \mathrm{MHz}_{\mathrm{CDCl}}\right) \delta: 8.98(\mathrm{~s}, 1 \mathrm{H}), 8.19(\mathrm{~s}, 1 \mathrm{H}, \mathrm{NH}), 8.07(\mathrm{~s}, 1 \mathrm{H}, \mathrm{NH}), 6.76(\mathrm{~s}, 1 \mathrm{H}), 4.43\left(\mathrm{~s}, 4 \mathrm{H}, \mathrm{OCH}_{2}\right), 2.71-$ $2.58(\mathrm{~m}, 8 \mathrm{H}), 2.13-2.06$ (m, 6H, Me), 2.00-1.90 (br.s, 4H), 1.68-1.60 (m, 4H), 1.55-1.46 (br.s, $2 \mathrm{H}) ;{ }^{13} \mathrm{C} \mathrm{NMR}(100$ $\mathrm{MHz}_{1} \mathrm{CDCl}_{3}$ ) $\delta: 167.5$ (C=O), 138.6, 137.4, 130.3, 129.8 (all C), 112.0 (2 x CH), $81.7\left(\mathrm{OCH}_{2}\right), 53.8,49.9$ (both $\mathrm{CH}_{2}$ ), $38.3(\mathrm{C}), 35.8,26.9$ (both $\mathrm{CH}_{2}$ ), $24.8(\mathrm{Me}), 24.0\left(\mathrm{CH}_{2}\right) ; \mathrm{HRMS}(\mathrm{ESI}) \mathrm{m} / z[\mathrm{M}-\mathrm{H}]^{-}$found 399.2397, $\mathrm{C}_{22} \mathrm{H}_{31} \mathrm{~N}_{4} \mathrm{O}_{3}$ requires 399.2396 .

$\mathbf{N}, N^{\prime}$-[2-(2-Oxa-7-azaspiro[3.5]nonan-7-yl)-5-(piperidin-1-yl)-1,4-phenylene]diacetamide (24). (0.386 g, 86\%); pale brown solid; $\mathrm{mp} 221-22{ }^{\circ} \mathrm{C} ; \mathrm{v}_{\max }\left(\right.$ neat, $\mathrm{cm}^{-1}$ ) 3362, 3286, 2941, 2869, 2808, 1672 (C=0), 1527, 1477, 
$1417,1367,1296,1239,1220,1195,1108,1062 ;{ }^{1} \mathrm{H} N M R\left(400 \mathrm{MHz}, \mathrm{CDCl}_{3}\right) \delta: 8.44(\mathrm{~s}, 1 \mathrm{H}, \mathrm{NH}), 8.33(\mathrm{~s}, 1 \mathrm{H}$, $\mathrm{NH}), 8.16(\mathrm{~s}, 1 \mathrm{H}), 8.11(\mathrm{~s}, 1 \mathrm{H}), 4.42\left(\mathrm{~s}, 4 \mathrm{H}, \mathrm{OCH}_{2}\right), 2.71(\mathrm{t}, J 5.1 \mathrm{~Hz}, 4 \mathrm{H}), 2.66(\mathrm{t}, J 5.1 \mathrm{~Hz}, 4 \mathrm{H}), 2.11(\mathrm{~s}, 3 \mathrm{H}, \mathrm{Me})$, 2.10 (s, 3H, Me), 2.01-1.87 (br.s, 4H), 1.68-1.59 (m, 4H), 1.57-1.46 (br.s, 2H); ${ }^{13} \mathrm{C} \mathrm{NMR}\left(100 \mathrm{MHz}^{\mathrm{CDCl}}\right)_{3} \delta$ : 168.0, 167.9 (both C=O), 139.3, 137.7, 129.4, 129.3 (all C), 112.0, 111.6 (both $\mathrm{CH}$ ), $81.7\left(\mathrm{OCH}_{2}\right), 53.7,49.9$ (both $\mathrm{CH}_{2}$ ), 38.3 (C), 35.9, 27.0 (both $\mathrm{CH}_{2}$ ), 24.9 (Me), $24.0\left(\mathrm{CH}_{2}\right) ; \mathrm{HRMS}$ (ESI) $\mathrm{m} / \mathrm{z}[\mathrm{M}+\mathrm{H}]^{+}$found 401.2536, $\mathrm{C}_{22} \mathrm{H}_{33} \mathrm{~N}_{4} \mathrm{O}_{3}$ requires 401.2553 .

Procedure for the synthesis of imidazobenzimidazoles 4 and 5. Diacetamide 21 or $\mathbf{2 4}$ (50 mg, $0.13 \mathrm{mmol}$ ) and Oxone $(0.250 \mathrm{~g}, 0.81 \mathrm{mmol})$ in $\mathrm{AcOH}(5 \mathrm{~mL})$ were stirred at $40{ }^{\circ} \mathrm{C}$ for $7 \mathrm{~h}$. The mixture was evaporated, $\mathrm{H}_{2} \mathrm{O}(10$ $\mathrm{mL}$ ) added, neutralized with solid $\mathrm{Na}_{2} \mathrm{CO}_{3}$, and extracted with $\mathrm{CH}_{2} \mathrm{Cl}_{2}(3 \times 5 \mathrm{~mL})$. The organic extracts were dried $\left(\mathrm{MgSO}_{4}\right)$, evaporated and recrystallized from EtOAc.

\section{6,7,11,12,13,14-Hexahydro-1H-2-oxa-7-azaspiro[3.5]nonan[1,2-a]pyrido[1',2':1,2]imidazo[4,5-f]benz-}

imidazole (4). (22 mg, 55\%); brown solid; $\mathrm{mp}$ (decomp. $>279^{\circ} \mathrm{C}$ ); $\mathrm{v}_{\max }$ (neat, $\mathrm{cm}^{-1}$ ) 3386, 2946, 2892, 1661, 1526, 1484, 1432, 1420, 1369, 1312, 1254, 1196, 1159, 1136, 1098; ${ }^{1} \mathrm{H} \mathrm{NMR}\left(400 \mathrm{MHz}^{\mathrm{C}} \mathrm{CDCl}_{3}\right) \delta: 7.90$ (d, J 0.9 $\mathrm{Hz}, 1 \mathrm{H}, 16-\mathrm{H}), 6.99(\mathrm{~d}, J 0.9 \mathrm{~Hz}, 1 \mathrm{H}, 9-\mathrm{H}), 4.54\left(\mathrm{~s}, 4 \mathrm{H}, 3,5-\mathrm{CH}_{2}\right), 4.08\left(\mathrm{t}, J 6.3 \mathrm{~Hz}, 2 \mathrm{H}, 7-\mathrm{CH}_{2}\right), 4.03(\mathrm{t}, J 6.1 \mathrm{~Hz}, 2 \mathrm{H}$, $\left.11-\mathrm{CH}_{2}\right), 3.34\left(\mathrm{~s}, 2 \mathrm{H}, 1-\mathrm{CH}_{2}\right), 3.05(\mathrm{t}, J 6.4 \mathrm{~Hz}, 2 \mathrm{H}), 2.43(\mathrm{t}, J 6.3 \mathrm{~Hz}, 2 \mathrm{H}), 2.14-2.06(\mathrm{~m}, 2 \mathrm{H}), 2.01-1.94(\mathrm{~m}, 2 \mathrm{H}) ;{ }^{13} \mathrm{C}$

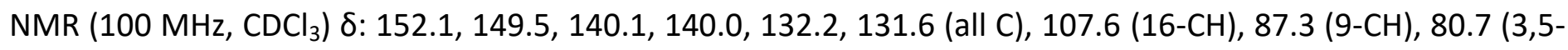
$\left.\mathrm{CH}_{2}\right), 42.6,38.9\left(7,11-\mathrm{CH}_{2}\right), 38.0(\mathrm{C}), 35.0\left(1-\mathrm{CH}_{2}\right), 30.8,25.7,22.7,20.8$ (all CH 2$) ; \mathrm{HRMS}$ (ESI) $\mathrm{m} / \mathrm{z}[\mathrm{M}+\mathrm{H}]^{+}$found 309.1720, $\mathrm{C}_{18} \mathrm{H}_{21} \mathrm{~N}_{4} \mathrm{O}$ requires 309.1715 .

\section{6,7,11,12,13,14-Hexahydro-1H-2-oxa-7-azaspiro[3.5]nonan[1,2-a]pyrido[1',2':1,2]imidazo[5,4-f]benzimid-} azole (5). (20 mg, 49\%); orange solid; $\mathrm{mp}$ (decomp. $>297^{\circ} \mathrm{C}$ ); $\mathrm{v}_{\max }$ (neat, $\mathrm{cm}^{-1}$ ) 2926, 2856, 1533, 1488, 1449, 1418, 1362, 1279, 1240, 1192, 1166, 1135, 1093; ${ }^{1} \mathrm{H}$ NMR $\left(400 \mathrm{MHz}, \mathrm{CDCl}_{3}\right) \delta: 7.53(\mathrm{~d}, J 0.8 \mathrm{~Hz}, 1 \mathrm{H}), 7.52(\mathrm{~d}, J$ $0.8 \mathrm{~Hz}, 1 \mathrm{H}), 4.64\left(\mathrm{ABq}, J 6.2 \mathrm{~Hz}, 4 \mathrm{H}, 3,5-\mathrm{CH}_{2}\right), 4.21\left(\mathrm{t}, J 6.3 \mathrm{~Hz}, 2 \mathrm{H}, 7-\mathrm{CH}_{2}\right), 4.16\left(\mathrm{t}, J 6.1 \mathrm{~Hz}, 2 \mathrm{H}, 14-\mathrm{CH}_{2}\right), 3.44(\mathrm{~s}$, $\left.2 \mathrm{H}, 1-\mathrm{CH}_{2}\right), 3.14(\mathrm{t}, J 6.4 \mathrm{~Hz}, 2 \mathrm{H}), 2.52(\mathrm{t}, J 6.3 \mathrm{~Hz}, 2 \mathrm{H}), 2.24-2.17(\mathrm{~m}, 2 \mathrm{H}), 2.10-2.04(\mathrm{~m}, 2 \mathrm{H}) ;{ }^{13} \mathrm{C} \mathrm{NMR}(100 \mathrm{MHz}$, $\mathrm{CDCl}_{3}$ ), 152.6, 150.0, 139.90, 139.85, 132.5, 132.0 (all C), 97.2, 97.0 (both $\mathrm{CH}$ ), $80.8\left(3,5-\mathrm{CH}_{2}\right), 42.6,38.9(7,14-$ $\mathrm{CH}_{2}$ ), 38.0 (C), $35.2\left(1-\mathrm{CH}_{2}\right.$ ), 30.8, 25.7, 22.7, 20.8 (all $\mathrm{CH}_{2}$ ); HRMS (ESI) $\mathrm{m} / z[\mathrm{M}+\mathrm{H}]^{+}$found $309.1721, \mathrm{C}_{18} \mathrm{H}_{21} \mathrm{~N}_{4} \mathrm{O}$ requires 309.1715 .

\section{Acknowledgements}

We gratefully acknowledge Kingston University for a PhD studentship for Darren Conboy. We thank Dr. Styliana I. Mirallai (NUI Galway) for HRMS analysis.

\section{Supplementary Material}

${ }^{1} \mathrm{H}$ and ${ }^{13} \mathrm{C}$ NMR spectra of novel compounds 4-6, 10-13, and 19-24, and ${ }^{1} \mathrm{H}$ NMR spectra of known compounds 14, 17 and 18 can be found in the Supplementary Material file. For spirocyclic oxetane ring-fused compounds $\mathbf{4}$ and $\mathbf{5}$ atom numbering is included, which is derived from the systematic compound names. 


\section{References}

1. Schulz, W. G.; Skibo, E. B. J. Med. Chem. 2000, 43, 629-638.

https://doi.org/10.1021/jm990210q

2. Suleman, A.; Skibo, E. B. J. Med. Chem. 2002, 45, 1211-1220.

https://doi.org/10.1021/jm0104365

3. Fagan, V.; Bonham, S.; Carty, M. P.; Aldabbagh, F. Org. Biomol. Chem. 2010, 8, 3149-3156.

https://doi.org/10.1039/c003511d

4. Fagan, V.; Bonham, S.; McArdle, P.; Carty, M. P.; Aldabbagh, F. Eur. J. Org. Chem. 2012, 1967-1975. https://doi.org/10.1002/ejoc.201101687

5. Fagan, V.; Bonham, S.; Carty, M. P.; Saenz-Méndez, P.; Eriksson, L. A.; Aldabbagh, F. Bioorg. Med. Chem. 2012, 20, 3223-3232.

https://doi.org/10.1016/i.bmc.2012.03.063

6. Zhang, K.; Chen, D.; Ma, K.; Wu, X.; Hao, H.; Jiang, S. J. Med. Chem. 2018, 61, 6983-7003.

https://doi.org/10.1021/acs.jmedchem.8b00124

7. Bull, J. A.; Croft, R. A.; Davis, O. A.; Doran, R.; Morgan, K. F. Chem. Rev. 2016, 116, 12150-12233. https://doi.org/10.1021/acs.chemrev.6b00274

8. Wuitschik, G.; Carreira, E. M.; Wagner, B.; Fischer, H.; Parrilla, I.; Schuler, F.; Rogers-Evans, M.; Müller, K. J. Med. Chem. 2010, 53, 3227-3246.

https://doi.org/10.1021/jm9018788

9. Conboy, D.; Mirallai, S. I.; Craig, A.; McArdle, P.; Al-Kinani, A. A.; Barton, S.; Aldabbagh, F. J. Org. Chem. 2019, 84, 9811-9818.

https://doi.org/10.1021/acs.joc.9b01427

10. Gurry, M.; McArdle, P.; Aldabbagh, F. Molecules 2015, 20, 13864-13874.

https://doi.org/10.3390/molecules200813864

11. Spiegel, L.; Kaufmann, H. Berichte der Dtsch. Chem. Gesellschaft 1908, 41, 679-685.

https://doi.org/10.1002/cber.190804101129

12. Sweeney, M.; Gurry, M.; Keane, L. A. J.; Aldabbagh, F. Tetrahedron Lett. 2017, 58, 3565-3567.

https://doi.org/10.1016/i.tetlet.2017.07.102

13. Gurry, M.; Sweeney, M.; McArdle, P.; Aldabbagh, F. Org. Lett. 2015, 17, 2856-2859.

https://doi.org/10.1021/acs.orglett.5b01317

14. Sweeney, M.; Keane, L. A. J.; Gurry, M.; McArdle, P.; Aldabbagh, F. Org. Lett. 2018, 20, 6970-6974.

https://doi.org/10.1021/acs.orglett.8b03135

15. Colomer, I.; Chamberlain, A. E. R.; Haughey, M. B.; Donohoe, T. J. Nat. Rev. Chem. 2017, 1, 0088. https://doi.org/10.1038/s41570-017-0088

16. Conboy, D.; Aldabbagh, F. Molbank 2020, 2020, M1118.

https://doi.org/10.3390/M1118

17. Nair, M. D.; Adams, R. J. Am. Chem. Soc. 1961, 83, 3518-3521.

https://doi.org/10.1021/ja01477a038

18. Meth-Cohn, O.; Suschitzky, H. Adv. Heterocycl. Chem. 1972, 14, 211-278.

https://doi.org/10.1016/S0065-2725(08)60954-X

19. Purkait, A.; Roy, S. K.; Srivastava, H. K.; Jana, C. K. Org. Lett. 2017, 19, 2540-2543.

https://doi.org/10.1021/acs.orglett.7b00832

20. Nguyen, T. B.; Ermolenko, L.; Al-Mourabit, A. Green Chem. 2016, 18, 2966-2970. 


\section{https://doi.org/10.1039/C6GC00902F}

This paper is an open access article distributed under the terms of the Creative Commons Attribution (CC BY) license (http://creativecommons.org/licenses/by/4.0/) 